\title{
Structure and kinetics in colloidal films with competing interactions
}

\author{
Mark D. Haw* \\ Department of Chemical and Process Engineering, University of Strathclyde, James Weir Building, \\ 75 Montrose Street, Glasgow G1 1XJ, United Kingdom
}

(Received 20 November 2018; published 7 January 2019)

\begin{abstract}
Using computer simulation we explore how two-dimensional systems of colloids with a combination of shortrange attractive and long-range repulsive interactions generate complex structures and kinetics. Cooperative effects mean the attractive potential, despite being very short-ranged compared to the repulsion, can have significant effects on large-scale structure. By considering the number of particles occupying a notional "repulsion zone" defined by the repulsion length scale, we classify different characteristic structural regimes in which the combination of attraction and repulsion leads to different structural and kinetic outcomes, such as compact clustering, chain labyrinths, and coexisting clusters and chains. In some regimes small changes in repulsion range and/or area fraction can change timescales of structural evolution by many orders of magnitude.
\end{abstract}

DOI: 10.1103/PhysRevE.99.012603

\section{INTRODUCTION}

Even simple interactions between colloidal particles can create surprisingly complex structures and kinetics, which is one reason for colloids' ubiquity and versatility in applications and their importance in generating new fundamental scientific insight, for example, in the physics of biological systems [1]. Colloidal structures and how they evolve over time are key to functionality in products such as foods, cosmetics, paints, coatings, lubricants, porous media, membranes, and filters. Structure and heterogeneity also determine materials' response to external forces in flow or under mechanical stress such as gravity. Hence such basic features of a product as the "shelf life" of a processed food or paint or the capture efficiency and selectivity of a porous filter are controlled fundamentally by the interaction potential between the constituent particles. Moreover while the equilibrium state is related to the interaction potential through thermodynamics, many useful systems are not at equilibrium, for example, metastable mixtures of oils and water in foods and personal care products: thus the structures created on the system's "journey" toward equilibrium, often different to those found at equilibrium, and how that journey can be interrupted, sometimes on very long timescales, i.e., the kinetics of structural change, are important factors. These kinetic aspects are even less straightforward to predict from the interaction potential.

It is well established that colloidal particles with purely attractive interactions can, depending on particle concentration, attraction strength, and attraction range, follow a range of "routes to equilibrium" involving liquid-gas phase separation, growth of compact clusters, and formation of fractal aggregates and space-filling gels [2,3]. In many practical examples of colloidal systems there is a more complex particle interaction composed of both attractive and repulsive contributions, at different length scales, and it remains less

\footnotetext{
*mark.haw@strath.ac.uk
}

clear how this combination of interactions controls structure and the kinetics of transit to equilibrium [4-10]. Generally, potentials featuring two length scales lead to complex microphase (equilibrium) separation and structural "crossovers" (see Ref. [11] for a recent study and related references). For two-dimensional (2D) systems, much previous simulation and theoretical work has shown how short-range attraction combined with longer-range repulsion, and even pure repulsion exhibiting multiscale features such as a "shoulder" at short length scale, can generate a complex plethora of structures in and out of equilibrium, including stripes, chains, clusters, and ordered crystals [12-14]. Similar patterns are seen in experimental systems such as magnetic particles and biological membranes [6,15-17] and have been discussed in the general context of geometrically frustrated materials [18]. 2D systems are particularly interesting for applications such as structured membranes, filters, films, coatings, and biological materials.

In this paper we focus on how short-range attraction and long-range repulsion in a simple colloidal model combine to determine structure and kinetic evolution on a range of length scales and timescales. We show that repulsion determines large-scale structure (the arrangement of structural "units" such as monomers, chains, chain labyrinths, ordered droplets and stripes, and lamellar-like polycrystals) by driving separation of the suspension into "structural units" of various types at the length scale defined by the repulsion range. Meanwhile the attraction can play a role at both short length scales (small-scale structural details) and length scales much larger than the attraction range, depending on the combination of attraction and repulsion. Under some conditions small changes in repulsion range and attraction strength can change kinetic timescales by many orders of magnitude. We show that a parameter combining the repulsion range and area fraction is useful to categorize regimes of different "structural units" in which the attractive interaction has qualitatively different effects on structure and kinetics. Thus a range of different structures can be realized and "captured" using repulsion 


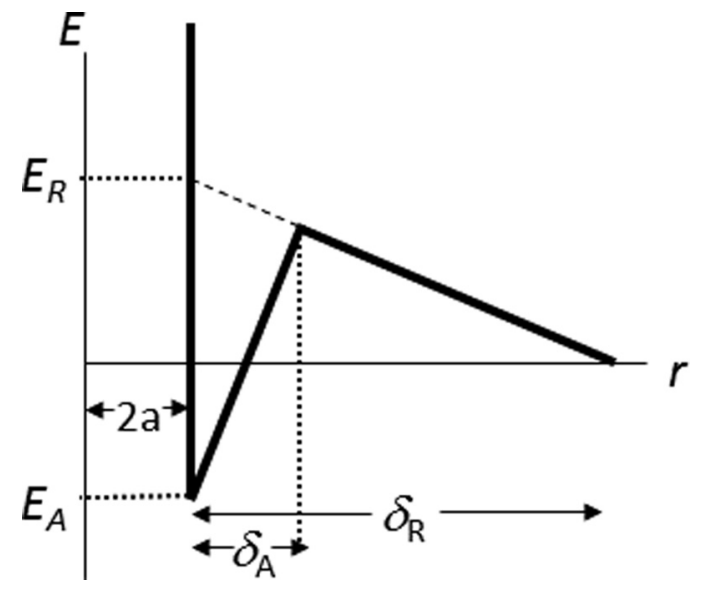

FIG. 1. Particle interaction potential as a function of the separation between particle centers, $r$, showing attraction and repulsion energies $E_{A}$ and $E_{R}$, respectively, and hardcore repulsion at center separation $r=2 a$ where $a$ is the particle radius. Attraction range $\delta_{A}$ and repulsion range $\delta_{R}$ are measured from the surface of the particle so that two particles begin to repel when their center separation is $r=\delta_{R}+2 a$.

and attraction between particles to change the structural path followed toward minimum energy.

\section{METHODS}

We use Monte Carlo simulations where a fixed area fraction $\Phi$ of monodisperse circular disk particles of radius $a$, interacting through a potential $U(r)$ (where $r$ is the interparticle center separation), diffuse in a square 2D box with periodic boundary conditions. For versatility of understanding the roles of attraction and repulsion, we employ a triangular piecewise potential. The particles have a hard-core repulsion for $r<2 a$ to prevent overlap, a triangular attractive potential with range $\delta_{A}$ and depth $E_{A}\left(E_{A}\right.$ is the potential at center separation $r=2 a$ ), and a longer-range triangular repulsive potential with range $\delta_{R}$. For convenience in the description of parameters here, we quantify the repulsion strength $E_{R}$ using what would be its effective value at $r=2 a$ in the absence of attraction, as shown in Fig. 1. Random-walk particle moves are selected from Gaussian distributions in dimensions $x$ and $y$ and accepted with probability $P=\exp (-\Delta E)$ where $\Delta E$ is the change in potential energy of the particle due to interactions with its neighbors associated with the proposed move. Particles therefore execute Brownian random walks within the interaction potential of their neighbors. We have checked that structures observed are insensitive to the mean step length used as long as this step length is a small fraction of the shortest range interaction (the attraction, in this case). Simulations are started from "fluid" configurations that are equilibrated by running the simulation with only hard-core repulsion (equivalent to high temperature) for a large number of steps until the measured pair correlation function $g(r)$ has reached a steady state, and thereafter immediately quenched to the required values of the energy parameters. In most data presented, the number of particles is $N=1000$ although we have checked for finite size effects using $N=4000$ in some cases, finding no change in results. The system box size in units of particle radius is determined by the required area fraction $\Phi$. Hereafter, for conciseness all parameters and results are quoted in appropriate reduced units: lengths (including interaction potential length scales) are given in units of the particle radius $a$, and energies are given in units of the thermal energy scale $k_{B} T$ where $k_{B}$ is Boltzmann's constant and $T$ the temperature. Since $k_{B} T$ is the typical scale of thermal fluctuation of a particle's kinetic energy, scaling interaction energies in this way allows a sense of how thermal fluctuation, which drives transit to equilibrium, competes with the interparticle interactions. Setting the interaction energy scale is thus equivalent to setting the inverse temperature. The mean step length of the particles' Brownian walk determines the timescale: for the value used here and a total run time of $10^{7}$ time steps (attempted moves per particle), for example, particles diffuse a distance (in units of $a$ ) similar to that which an isolated $1 \mu \mathrm{m}$ diameter colloid in water at room temperature would diffuse in $1 \mathrm{~s}$, equivalent to a small multiple of the particle radius $a$. While particles therefore do not diffuse far over the reported run times, the area fractions and interaction ranges considered here mean that characteristic structures are formed quickly, i.e., without substantial particle diffusion. This has implications for whether systems reach thermodynamic equilibrium: as results will show and as discussed below, most systems are trapped by the strong cooperative interactions in nonequilibrium structures that evolve very slowly.

\section{RESULTS}

In the present work, we focus on the roles of $\delta_{R}, \Phi$, and $E_{A}$, maintaining a constant attraction range $\delta_{A}=0.05$ and a constant repulsion energy (at zero separation) $E_{R}=45$. Thus we focus here on strongly repulsive systems, although as we shall see the attractive part of the interaction turns out to have significant effects despite its short length scale. When repulsive interactions are dominant, we expect particles to disperse individually so as to minimize global repulsion. But this is possible only as long as the combination of area fraction $\Phi$ and repulsion range $\delta_{R}$ gives space enough for all individual particles to separate beyond $\delta_{R}$. The boundary of this singleparticle dispersed phase, in terms of repulsion range and area fraction, can be estimated by assuming that repulsion drives the suspension to separate into groups of particles occupying "repulsion zones" of radius $1+\delta_{R} / 2$ (in units of particle radius). At area fraction $\Phi$, the average number of particles contained in each such zone is $n_{b}=\Phi\left(1+\delta_{R} / 2\right)^{2}$. For $n_{b} \leqslant$ 1 there is space for monomers to minimize repulsion by occupying separate zones, remaining individually dispersed. At $n_{b}>1$ repulsion zones must contain multiple particles and the configuration of particles that would minimize free energy (in equilibrium) or indeed nonequilibrium configurations that may appear and evolve as the system journeys toward equilbrium are less clear. As we will see $n_{b}$ is a useful parameter to categorize and contrast structure and the kinetics of evolution toward equilibrium under different conditions.

In Fig. 2 we plot snapshots of the particle configurations in a "phase diagram" of $n_{b}$ versus $\Phi$ at a fixed value of $E_{A}=-7$ and fixed number of time steps $t=10^{6}$ after the quench to the given values of the interaction parameters. As 


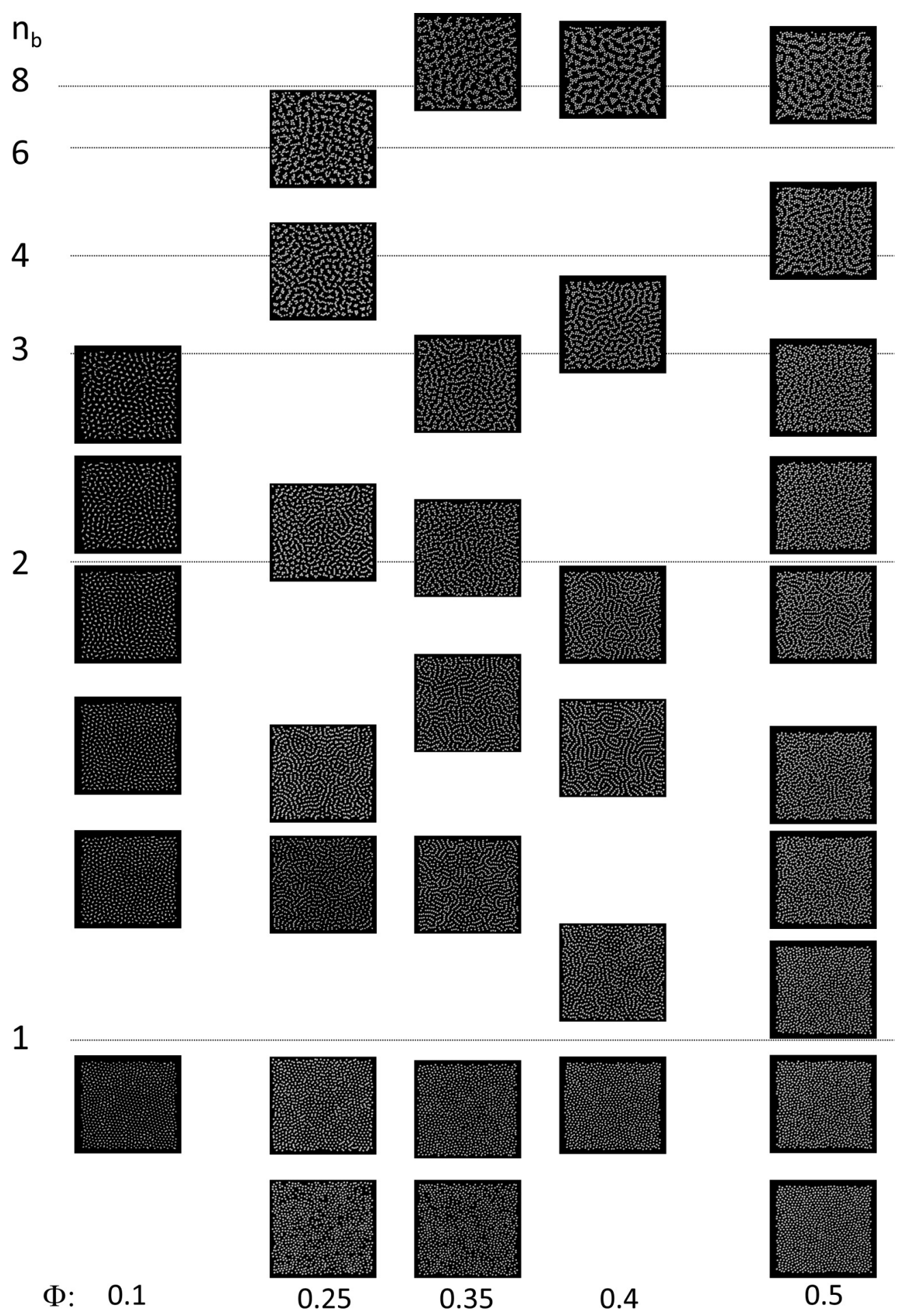

FIG. 2. Simulation snapshots at $t=10^{6}$ time steps and $E_{A}=-7$ showing dependence of structure on area fraction $\Phi$ and number of particles per "repulsion zone," $n_{b}$ (see text). Particles are plotted as disks with radius $a$.

can be seen the boundary $n_{b} \simeq 1$ predicts reasonably well the limit of the single-particle dispersed phase: typically, just below $n_{b}=1$, snapshots show systems mostly dispersed as single particles, with a small population of dimers and trimers. Once $n_{b}$ is somewhat larger than 1, particles can no longer minimize repulsion by individually separating to interparticle distances greater than $\delta_{R}$ and must "choose" another structural strategy. For example, some particles might group closer to enable others to get further apart, to reach overall minimum free energy. Figure 2 shows that various structural types are observed depending on distance above the $n_{b}=1$ boundary, including chains, intertwined "labyrinths," mixtures of chains and small clusters, quasiordered compact groups, and stripes. (Results for different attraction $E_{A}$ show similar $n_{b}$ boundaries between characteristic structures, although as we show later, $E_{A}$ has significant effect on the later time kinetic aging 

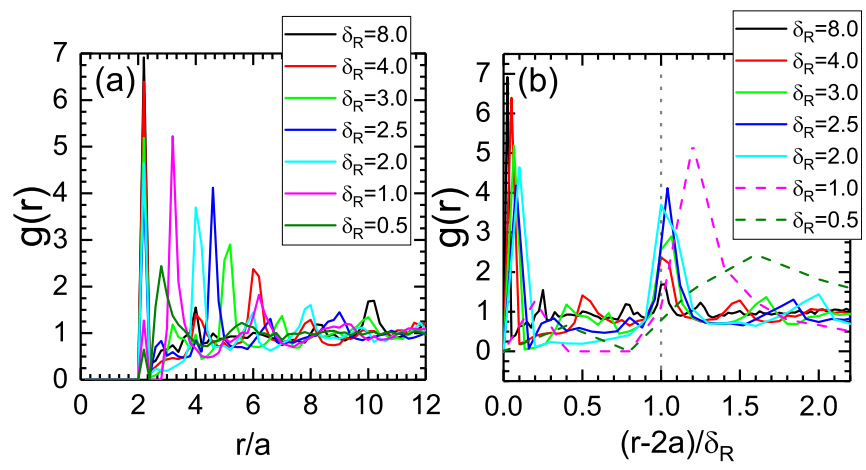

FIG. 3. (a) Pair correlation function $g(r)$ (with $r$ scaled by the particle radius $a$ ) for example simulations at $t=10^{6}$ time steps, with $\Phi=0.35, E_{A}=-7$ and a range of $\delta_{r}$ values. (b) $g(r)$ vs the particle surface separation $r-2 a$ scaled by $\delta_{R}$. Dashed lines show data for $n_{b} \leqslant 1$. Systems with $n_{b} \gtrsim 1$ feature a strong peak at $(r-2 a) / \delta_{R}=$ 1 (dotted vertical line).

of the systems.) Plotting the pair correlation function $g(r)$ versus the particle surface separation $(r-2)$ scaled by $\delta_{R}$ [Fig. 3(b)] produces a strong peak at $(r-2) / \delta_{R}=1$ for all cases above $n_{b} \simeq 1$. Hence for $n_{b} \gtrsim 1$ the repulsion range $\delta_{R}$ sets the dominant "interstructural" length scale, i.e., the separation between chains, groups, etc., while for $n_{b}<1$ particles have enough space to separate at distances greater than the repulsion range, so that $\delta_{R}$ is less of a determining scale.

In fact, such order characterized by $\delta_{R}$ occurs with or without short-range attraction (at this high value of $E_{R}$ at least), showing that ordered structures such as chains, groups, and stripes do not require an attractive interaction and are simply driven by repulsion [13]. The repulsive interaction drives separation into characteristic structural units separated by length scale $\delta_{R}$. The strong repulsive interactions mean that individual groups are relatively stable, i.e., there is only slow "swapping" of particles between groups (see below for further examination of the role of thermal aging). We shall see, however, that the attractive part of the interaction can have significant effects on the structural details at both long and short length scales and on the time evolution of the system.

As demonstrated by Fig. 2 the type of structural unit formed depends on how many particles must occupy the repulsion zone, i.e., $n_{b}$. First, we discuss behavior at large $n_{b} \gg 1$. Structures here are typified by groups of particles, groups being dispersed and roughly ordered on the $\delta_{R}$ length scale (Fig. 4). One can think of this as an effective rescaling: instead of dispersed, ordered single particles at $n_{b} \leqslant 1$, at large $n_{b}$ free energy is minimized by separation into dispersed, ordered larger groups of particles. Locally, particles within each group repel each other, but globally the separation from neighboring groups means an overall reduction in free energy.

Being short-ranged, the attractive part of the potential does not affect the intergroup length scale, but it does change the local group structure, the way the groups "connect" to each other, and the kinetics of structural evolution (compare the different attraction strength examples in Fig. 4). Strong attraction [Figs. 4(a) and 4(b)] leads to more disordered group shapes and, at early enough times, more chainlike structure,
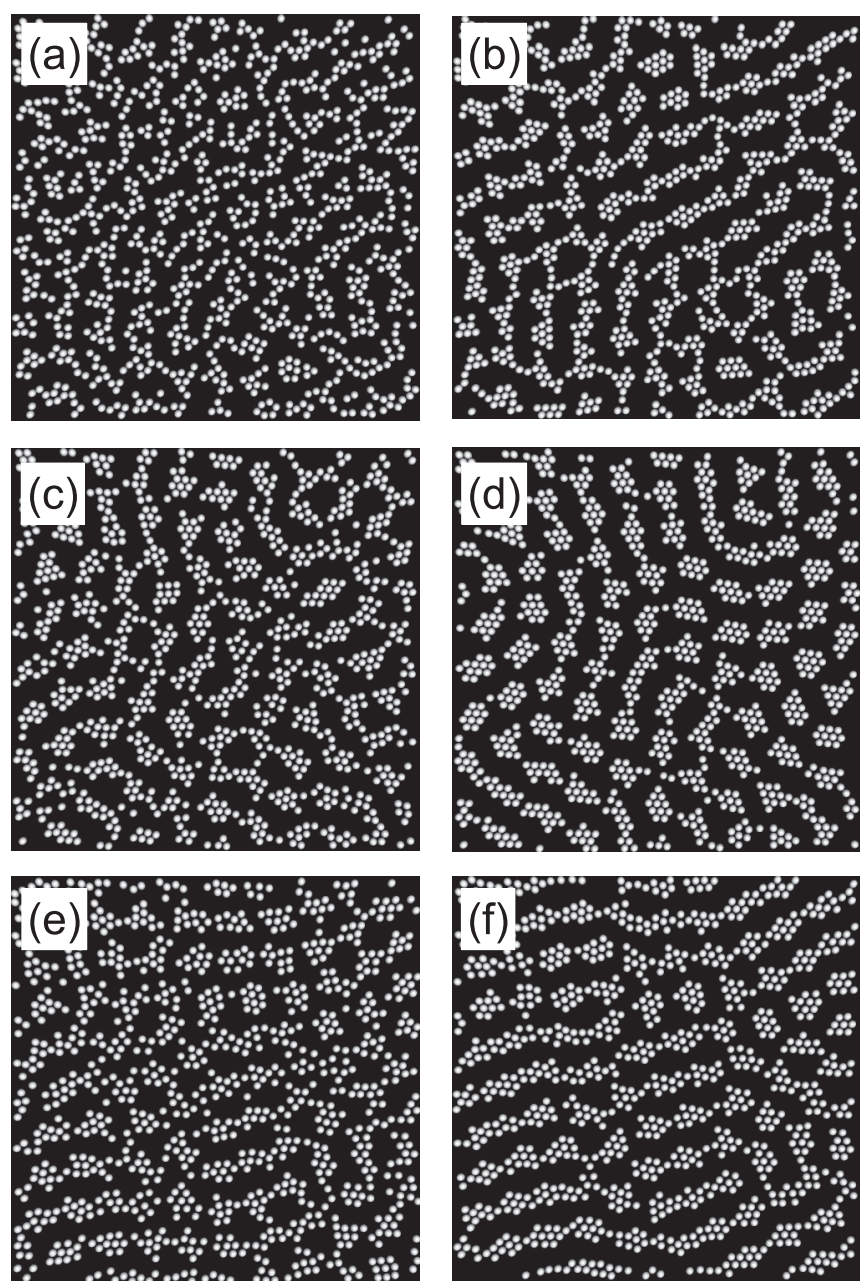

FIG. 4. Snapshots from simulations for $\Phi=0.35$ and $\delta_{R}=8.0$, for $t=10^{6}$ (left column) and $t=10^{7}$ (right column) time steps, contrasting the role of attraction energy $E_{A}$ : (a, b) $E_{A}=-7$; (c, d) $E_{A}=41$; (e, f) $E_{A}=45$, i.e., $E_{A}=E_{R}$ implying no attractive "well" in the interparticle potential.

which compactifies over time due to thermal fluctuations. Weaker attraction [Figs. 4(c) and 4(d)], allowing increased rates of thermal fluctuation, leads to better defined regular groups. Removing attraction altogether ]Figs. 4(e) and 4(f)] allows groups to connect and form structures similar to stripes, although there remains strong long-range ordering (stripe separation) and remnants of group structure along the stripes.

A measure based on the scale of void spaces opened up by the interaction provides more quantitative information on the roles of repulsion and attraction. We identify for each particle the region of void space closer to it than to any other particle, i.e., the particle's Voronoi cell, and record for each particle the distance from the particle surface to the most distant point in its Voronoi cell, $R_{\max }$. $R_{\max }$ identifies particles bordering large voids compared to particles in the bulk of compact clusters or groups [see Fig. 5(e) for an example snapshot]. The distribution of $R_{\max }$ across the population of particles, and how this changes with time, distinguishes different cases of structure and kinetic evolution depending on repulsion range and attraction energy [Figs. 5(a)-5(d)]. Peaks in the 

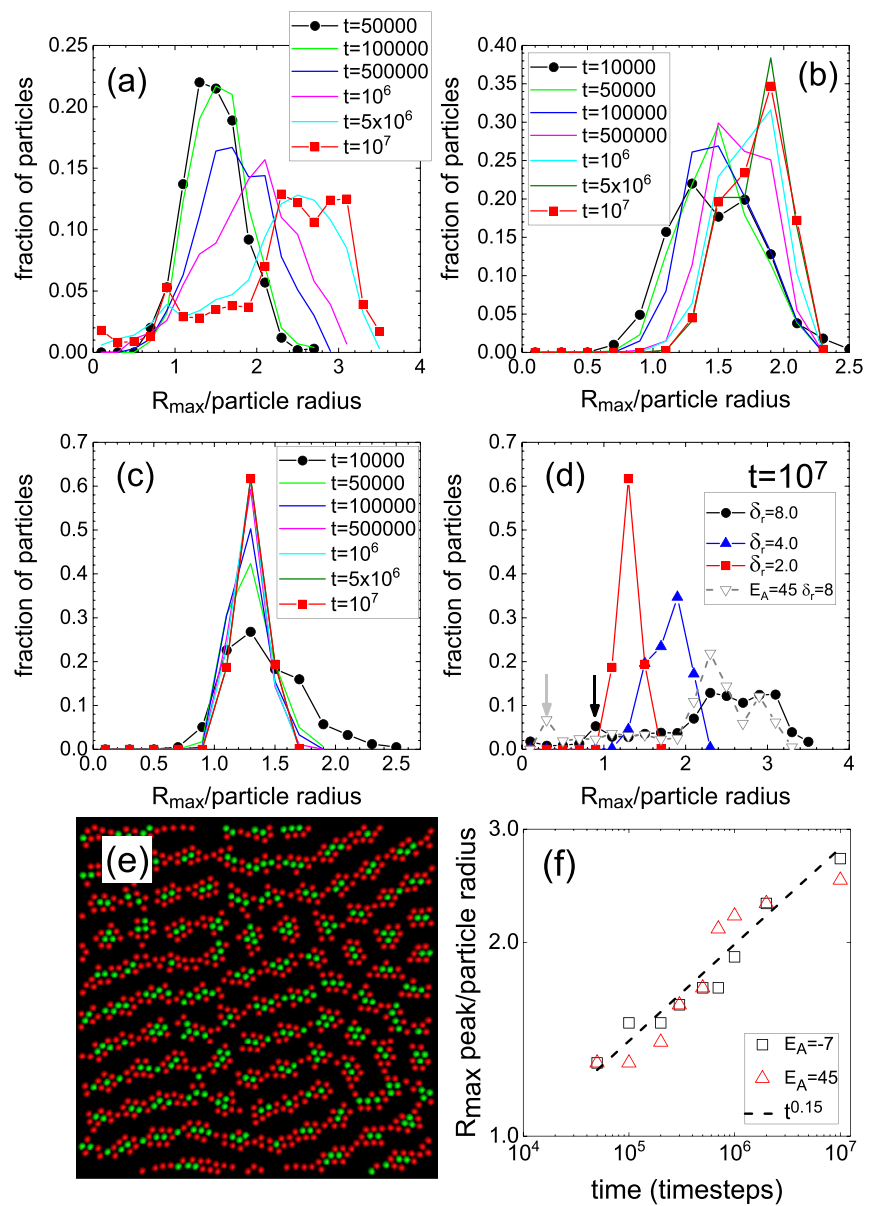

FIG. 5. Distributions of the distance to the most distant point in a particle's Voronoi cell, $R_{\max }$, from simulations with $\Phi=0.35, E_{A}=$ -7 at (a) $\delta_{R}=8.0\left(n_{b}=8.75\right)$; (b) $\delta_{R}=4.0\left(n_{b}=3.15\right)$; (c) $\delta_{R}=$ $2.0\left(n_{b}=1.4\right)$; (d) late-time $\left(t=10^{7}\right.$ time steps $)$ distributions at different $\delta_{R}$, and, for comparison, the distribution for $\delta_{R}=8.0$ and $E_{A}=45$; (e) example simulation snapshot $\left(\delta_{R}=8.0, E_{A}=45, t=\right.$ $10^{7}$ ) with particles with $R_{\max }<1.5$ in green, $R_{\max } \geqslant 1.5$ in red; (f) time dependence of the length scale indicated by the large-distance peak in the $R_{\max }$ distribution for $\delta_{R}=8.0$, for both $E_{A}=-7$ and $E_{A}=45$. The dashed line illustrates a power law with exponent of 0.15 .

distribution at a given size indicate significant populations of voids of that size. The presence of multiple peaks indicates that there is more than one characteristic "environment" for particles in the system, in other words there are regions with different local void geometries and interparticle structures, such as particles inside and particles at the surface of groups.

At $n_{b} \gg 1$ [e.g., $\delta_{R}=8, \Phi=0.35$ giving $n_{b}=8.75$; Fig. 5(a)] an early-time broad peak in the $R_{\max }$ distribution transforms into a peak traveling to larger $R_{\text {max }}$, demonstrating the growth of large intergroup voids of a characteristic size, and a small peak at low $R_{\max }$, corresponding to particles inside compact groups. Plotting the position of the large $R_{\max }$ peak over time for different values of $E_{A}$ [Fig. 5(f)] reveals power law kinetics of group separation (void growth) with an exponent $(\cong 0.15)$ independent of $E_{A}$. In other words at $n_{b} \gg$ 1 the structure evolves toward a state of quasiordered groups at all $E_{A}$, driven by growth of voids whose characteristic
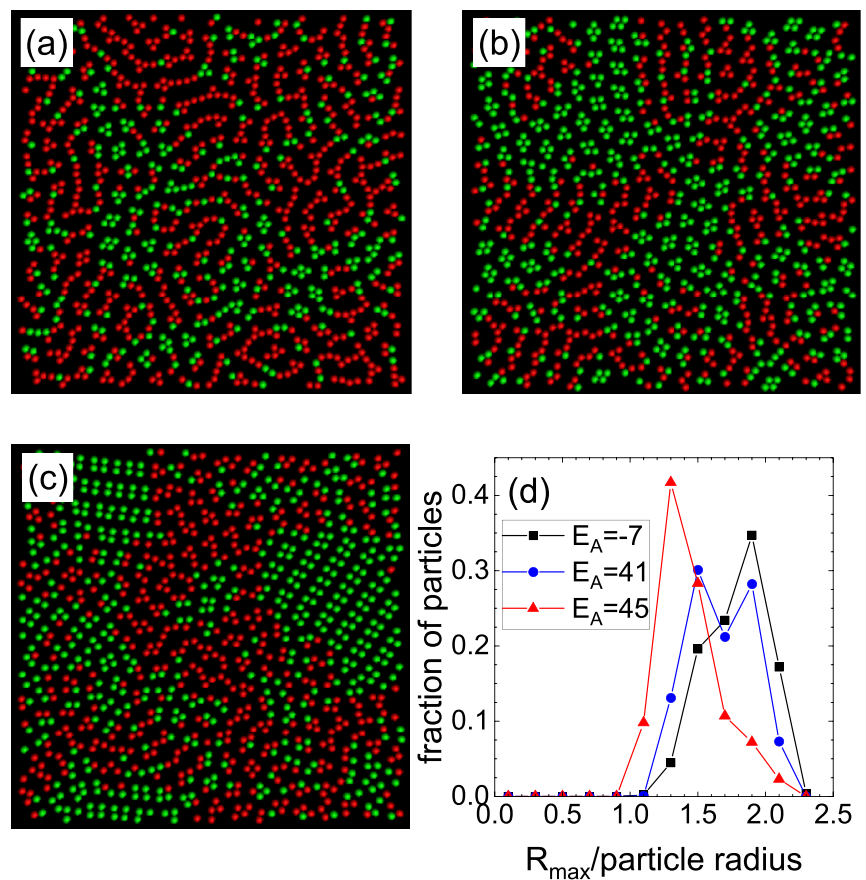

FIG. 6. (a-c) Simulation snapshots for $\Phi=0.35$ and $\delta_{R}=$ $4.0\left(n_{b}=3.15\right)$ at $t=10^{7}$ time steps. Particles with $R_{\max }<R_{M}$ are plotted in green, particles with $R_{\max } \geqslant R_{M}$ in red, where $R_{M}$ is selected to divide the $R_{\max }$ distributions (see panel d) into two halves. (a) $E_{A}=-7, R_{M}=1.7$; (b) $E_{A}=41, R_{M}=1.7$; (c) $E_{A}=45$, $R_{M}=1.4$. (d) Late-time $\left(t=10^{7}\right.$ time steps) $R_{\max }$ distributions at the different $E_{A}$ corresponding to the snapshots in panels (a) to (c).

size obeys an $E_{A}$-independent growth law. At large $n_{b}$ phase separation into voids and clusters is therefore dominated and driven by repulsion (at least at the relatively strong repulsion studied, $E_{R}=45$ ). Nevertheless the details of the $R_{\text {max }}$ distribution and hence the finer details of structure do still depend on $E_{A}$. Interestingly, the low- $R_{\max }$ peak at late time [arrows in Fig. 5(d)] is at significantly smaller length scale for $E_{A}=45$ compared to $E_{A}=-7$. At first sight this implies, perhaps surprisingly, that local structure inside groups of particles seems more compact at weaker attraction. Further study of the detailed local structure will be reported elsewhere, but for now we point out that increased regularity, rather than compactivity, of the local structure inside a group will tend to reduce $R_{\text {max }}$, while particle aggregation at stronger attraction will tend to produce a more irregular structure with larger spaces and hence larger $R_{\text {max }}$, since stronger attraction makes local restructuring more difficult.

Next we turn to intermediate $n_{b}$ [e.g., $\delta_{R}=4, \Phi=0.35$ giving $n_{b}=3.15$, Fig. 5(b)], where phase separation structure and kinetics are more complex and, furthermore, depend more substantially on the attraction energy $E_{A}$. At zero attraction ( $E_{A}=E_{R}=45$ ) some particles form small ordered groups, themselves arranged in layers reminiscent of lamellae; but this phase exists in polycrystal domains within a more disordered phase [Fig. 6(c)]. At strong attraction, by contrast, regions of small compact groups (typically of four particles) coexist with regions of short roughly aligned chains [Fig. 6(a)]. Particles in the ordered units and compact groups have lower $R_{\max }$ while 


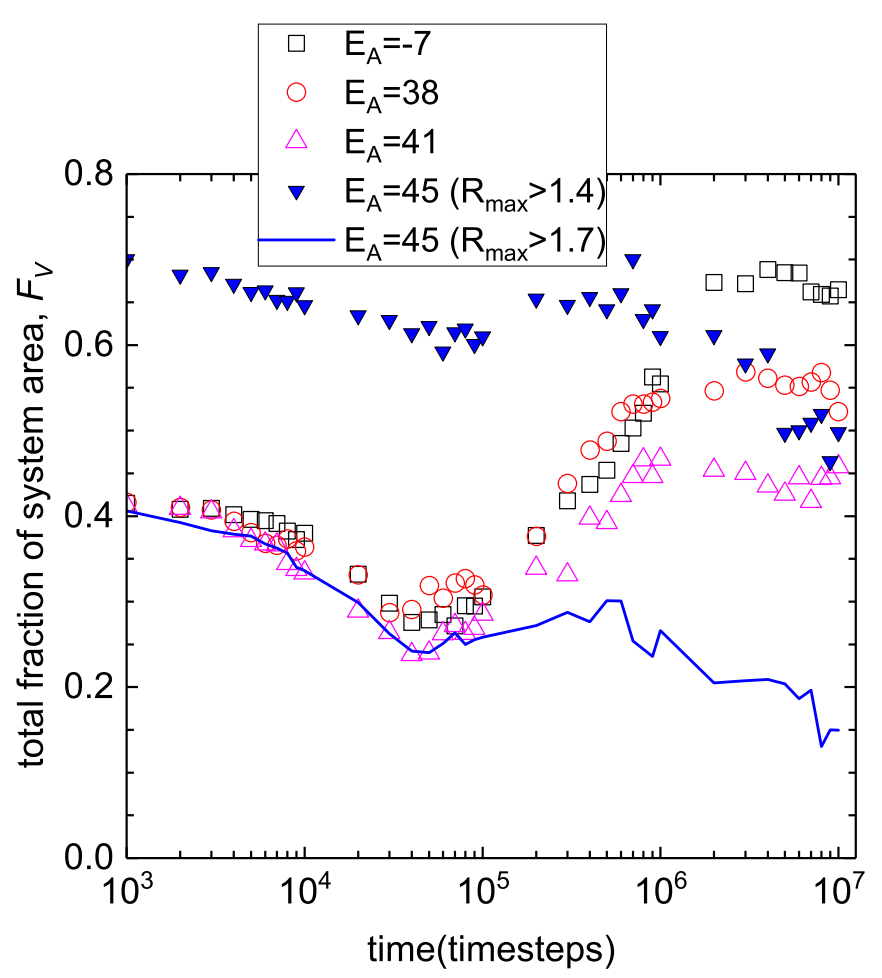

FIG. 7. Time dependence of $F_{V}$, the fraction of the system area occupied by particles adjacent to "large" voids, i.e., particles with $R_{\text {max }} \geqslant R_{M}$, for $\Phi=0.35$ and $\delta_{R}=4.0\left(n_{b}=3.15\right)$ at various $E_{A}$. For $E_{A}=-7,38$ and 41 we use $R_{M}=1.7$, while for $E_{A}=45$, $R_{M}=1.4$, corresponding to the snapshots in Fig. 6. For $E_{A}=45$ we also show $R_{M}=1.7$ (the solid line) for comparison.

particles in the still disordered and short aligned chain part of the system have higher $R_{\max }$ as shown by the different colors in Fig. 6. When the number of particles that must be fitted into the local repulsion zone $n_{b} \approx 3$, the possible structural responses are evidently more complex.

The kinetics of structural evolution in such a coarsening system can be tracked over time by computing $F_{V}$, the fraction of system area occupied by particles in the large $R_{\text {max }}$ regions i.e., particles with values of $R_{\max }$ above a characteristic value $R_{M}$ distinguishing the two coexisting regions. We compute $F_{V}$ simply by adding the Voronoi cell areas of particles with $R_{\text {max }} \geqslant R_{M}$ (Fig. 7). $R_{M}$ is chosen to split the $R_{\text {max }}$ distribution approximately evenly and most clearly separate the system into different characteristic structures above and below $R_{M}$, as demonstrated by the snapshots in Fig. 6. As shown for one example in Fig. 7 changing $R_{M}$ shifts the $F_{V}$ data in absolute terms but does not change the form of the temporal dependence. For all $E_{A}$ the total area of the disordered large void phase $F_{V}$ initially decreases before, at similar time for all $E_{A}$, beginning to rise. However, without attraction $\left(E_{A}=45\right)$ $F_{V}$ subsequently decreases again and is still decreasing at the longest run times we reach: the area occupied by crystallites of lamellae-arranged ordered units, associated with low $R_{\max }$, continues to fill an increasing fraction of the system over time. In contrast, with increased attraction strength, after the initial decrease $F_{V}$ continues to increase to a maximum at an $E_{A}$-dependent value: large $R_{\max }$ (void) regions form and fill an increasing fraction of the system up to a limit that depends on the strength of attraction. Having reached this limit the void regions persist, the structure showing no evidence of approaching back to the no-attraction case. At intermediate $n_{b}$ it seems therefore that attraction enables generation and freezing of larger voids into the system, leaving a long-lasting structure different from the no-attraction case. Even a weak attraction of $E_{A}=41$ (i.e., $E_{R}-E_{A}=4 k_{B} T$ ) is enough both to significantly change the structure and to maintain this change against thermal restructuring [Fig. 6(b)].

Given that the attractive interaction is very short-ranged compared to the scale of voids it is surprising that it should have such large-scale effects. We propose that this is due to a cooperative effect of repulsion and attraction that evidently becomes important for intermediate $n_{b}$. Repulsion, being long-ranged, drives the separation of the system, generating large $R_{\max }$ voids. However during this process, the particles bordering these voids form chains. These chains, and thus the large void regions, are stabilized by the combination of repulsion (chains aligning approximately parallel to minimize repulsion) and attraction (holding neighbors in the chains together against local fluctuations that would otherwise convert short chains to small compact units) [14]. The result is a coexistence of zones of short aligned chains and zones of small compact groups as seen in Figs. 6(a) and 6(b). At zero attraction $\left(E_{A}=E_{R}\right)$, however, chains are not maintained by attraction between neighbors, and thus there is no interchain repulsion to stabilise the large-void fraction of the system. At intermediate $n_{b}$, therefore, competition between different structural processes leads to complex long-lasting structural differences at different attraction strength.

For $1.0<n_{b} \leqslant 2.0$ a distinctly different characteristic structure is observed, the so-called "chain labyrinth" (Fig. 8). Here the $R_{\max }$ distribution is sharply single-peaked [Fig. 5(c)]: almost all particles are in tightly intertwined correlated chains and hence at the edge of large voids. Figure 8(a) shows that effective "labyrinths" still form even at low particle concentration: the chains of the labyrinth are shorter, even reduced to dimers and trimers, but their mutual repulsion still leads to an interwoven labyrinthine arrangement. For $n_{b}$ between 1 and $2, \simeq 2$ particles occupy a typical repulsion zone and by definition will form a dimer with a defined axis. When repulsion is long-ranged enough, a particle approaching such an existing dimer feels minimum repulsion approaching along the axis of the chain compared to perpendicular, so that any growth will tend to promote chains. Meanwhile minimization of repulsion between different neighboring chains will encourage local parallel alignment of growing chains. Hence free energy will be reduced by the formation of chains [14].

Because chains are stabilized by interchain repulsion, attractive interactions are not required for the chain labyrinth phase to form. The details of the local structure do, however, depend on $E_{A}$. Strong attraction freezes in local structure, e.g., the long, straight parallel chains of Fig. 8(e); reducing attraction allows increasingly more chain "flexibility" and curvature [Figs. 8(f) and 8(g)]. From an applications point of view, changing $E_{A}$ could therefore be used to change the shape and arrangement of holes in a quasi-2D system such as a colloidal film or coating, perhaps useful for designing selective filters and membranes or patterned surfaces. 

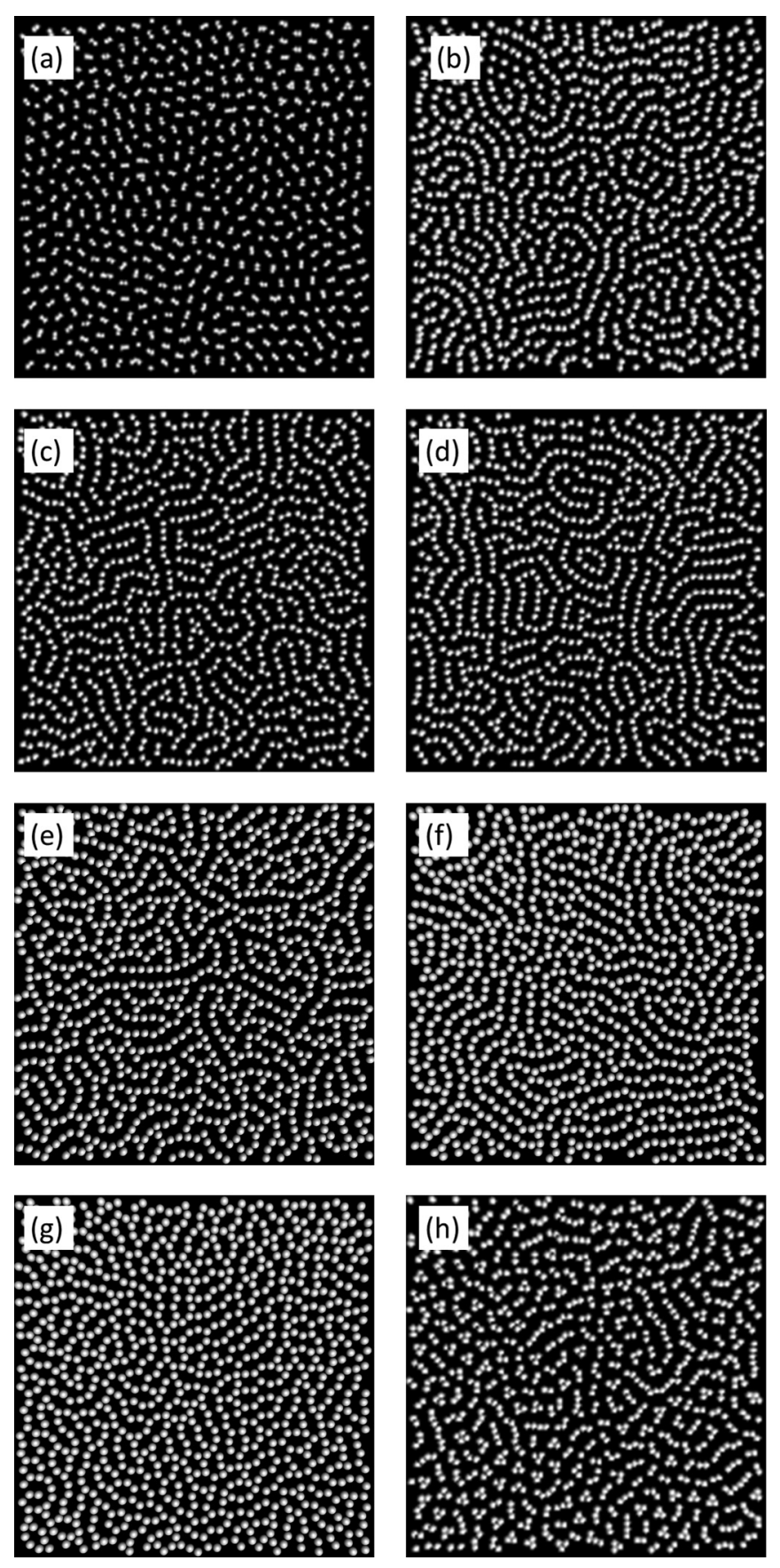

FIG. 8. Simulation snapshots showing chain labyrinth structure at $t=10^{6}$ time steps (a-d) and $t=5 \times 10^{6}$ time steps (e-h) for a range of $\Phi$ and energy parameters as follows: (a) $\Phi=0.1$, $E_{A}=-7, \delta_{R}=6.0\left(n_{b}=1.6\right) ;\left(\right.$ b) $\Phi=0.25, E_{A}=-7, \delta_{R}=$ $3.0\left(n_{b}=1.56\right)$; (c) $\Phi=0.35, E_{A}=-7, \delta_{R}=2.5\left(n_{b}=1.77\right)$; (d) $\Phi=0.4, E_{A}=-7, \delta_{R}=2.0\left(n_{b}=1.6\right)$; (e) $\Phi=0.5, E_{A}=$ $-7, \delta_{R}=2.0\left(n_{b}=2.0\right)$. (f) $\Phi=0.5, E_{A}=41, \delta_{R}=2.0\left(n_{b}=\right.$ 2.0); (g) $\Phi=0.5, E_{A}=45, \delta_{R}=2.0\left(n_{b}=2.0\right)$; (h) $\Phi=0.25$, $E_{A}=-7, \delta_{R}=4.0\left(n_{b}=2.25\right)$, showing onset of coexistence of chains and small compact groups.

As $n_{b}$ is increased beyond $\simeq 2$ the chain labyrinth begins to give way to mixtures of chains and small groups such as triplets [Fig. 8(h)]. As more than two particles (on average) need to occupy the repulsion zone in order to achieve separation at the $\delta_{R}$ length scale, we therefore expect a mixture of zones containing triplets (local $n_{b}=3$ ) and zones containing chains (local $n_{b}=2$ ).

An important question for design of functional materials that are not at thermodynamic equilibrium (see below for further discussion) is the kinetic stability or otherwise of different structures. We examine structural conversion between monomers, chains and more compact structures by identifying particles as belonging to chains or compact groups as follows. A chain particle will have at least two neighbors (particles within some cutoff distance) but, crucially, to qualify as a chain particle these neighbors must not be neighbors of each other. (Here we use a cutoff distance of $0.5 a$ to define neighbors: we have checked that results are insensitive to this as long as it is not too large, i.e., does not approach the repulsion range. Note that such neighbors in chains are not therefore necessarily bonded by attractive interactions: we seek a general structural analysis that can be applied even for zero attraction, important because we know that chains form even with zero attraction.) Particles at the end of chains, which have only one neighbor, are also identified as chain particles if that neighbor is itself a chain particle. Conversely we define a group particle as having at least two neighbors which are also neighbors of each other. Finally there are also particles with no neighbors within the cutoff distance, i.e., monomers. Inspection of snapshots (see the inset to Fig. 9 for an example) is used to validate that this method successfully distinguishes between chains, groups, and monomers.

The temporal behavior of the fractions of particles in chain and group configurations (Fig. 9) demonstrates that in the chain labyrinth regime $\left(1.0<n_{b} \leqslant 2.0\right)$ conversion from chain to group is very slow, the growth of the group fraction being approximately logarithmic in time. Chain labyrinths persist for long times. Even though coarsening would normally be seen as a local process depending on the shortrange interaction of two particles, with an Arrhenius-type characteristic rate determined simply by the attractive interaction, because the particles are assembled into chains and neighboring chains are strongly correlated at the significantly larger length scale $\delta_{R}$ by the repulsion, coarsening, e.g., into groups requires not just breaking local attractive bonds but overcoming the repulsion of neighboring parallel chains: a much more energetically costly disturbance in the local energy balance.

Figure 9 shows that there is a "maximum chain" state at a particular $n_{b}=n_{\max }$ where the fraction of particles in groups and monomers is minimized. In Fig. $9, \Phi=0.35$, for example, this occurs around $n_{\max } \approx 1.4$. For $n_{b}$ below this, we see chain-monomer coexistence. The observed monomers tend to be grouped in lines akin to chains: these monomers are the "remnants" of the ordered single-particle dispersed phase. As $n_{b}$ increases above $n_{\max }$ the population of particles in groups increases, until by $n_{b} \simeq 2.0$ significant numbers of particles exist in groups rather than chains.

The fact that the proportion of particles in chains and groups is still changing at the longest run times demonstrates that the systems are not at thermodynamic equilibrium. Restructuring and aging processes are in fact occurring on a range of timescales and length scales. At early times after the quench from zero interaction to the given value of $E_{R}$ and $E_{A}$, 


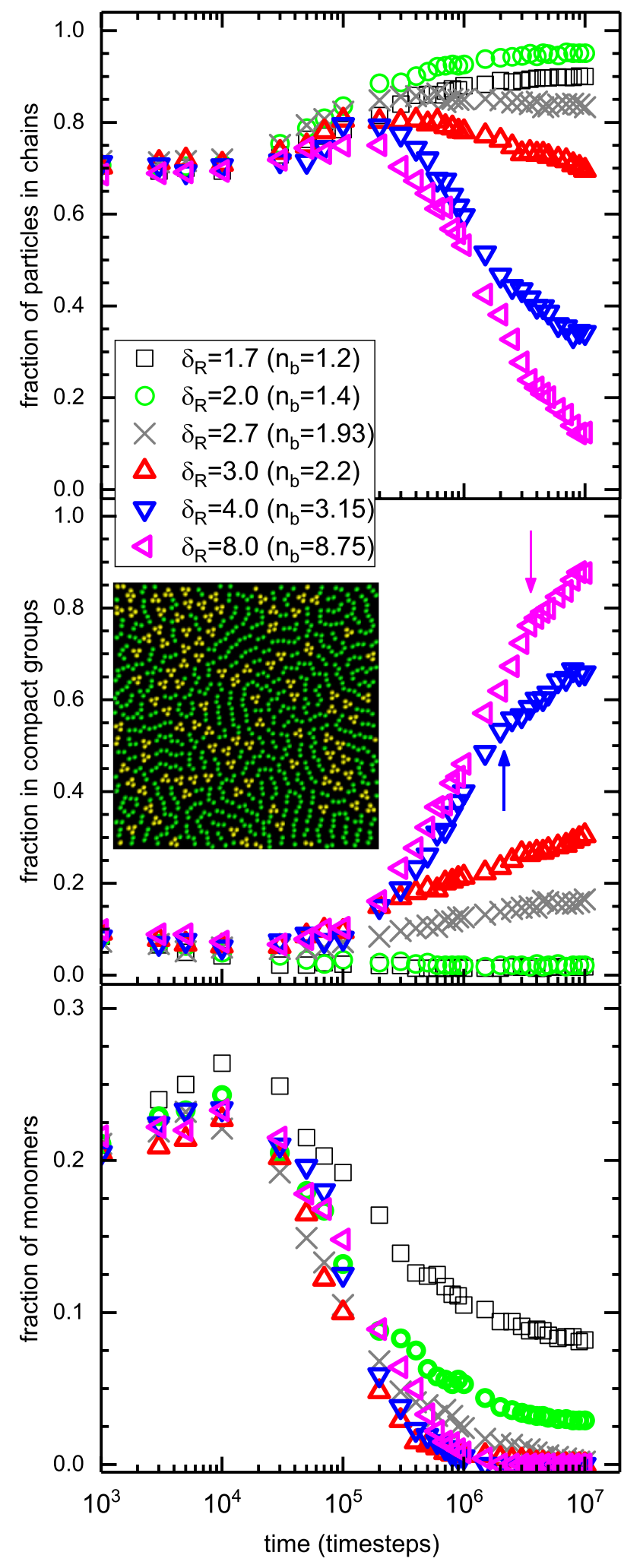

FIG. 9. Fraction of particles belonging to chains (top), compact groups (middle), and fraction of monomers (bottom), for $\Phi=0.35$, $E_{A}=-7$, and various $\delta_{R}\left(n_{b}\right)$. The inset to the middle plot shows an example snapshot $\left(\Phi=0.35, E_{A}=-7, \delta_{R}=3.0, n_{b}=2.2\right)$ at $t=$ $10^{7}$ time steps, with particles belonging to chains plotted in green, particles in groups in yellow. Arrows in the middle plot indicate change in growth of fraction in compact groups, as discussed in the text.
Fig. 9(a) shows that the majority of particles exist in chains. Thermal conversion over time from chains to more stable triplets and larger groups occurs at a rate that increases as $n_{b}$ increases from $n_{\max }$. At large $n_{b}$ the rate of conversion also reduces at later time [see arrows in Fig. 9(b) for $n_{b}=3.15$ and $\left.n_{b}=8.75\right]$. This switch in growth rate coincides with when $F_{V}$, the fraction of system area contributing to large voids, reaches its maximum value (Fig. 7). The increase in $F_{V}$ indicates growth of large voids between chains or groups as particles seek reduced energy states: $F_{V}$ reaches a constant as this route to reducing repulsion energy by maximizing separation reaches a limit when there is no further space to create large voids. According to Fig. 9(b), local chain-togroup reconfiguration continues after this time, representing a secondary route to further minimizing energy but with a different (slower) characteristic timescale.

The magnitude of restructuring rate is highly dependent on $n_{b}$ : for example, from Fig. 9 at $n_{b}=3.15\left(\delta_{R}=4\right)$ the fraction of particles in groups reaches 0.5 in around $10^{6}$ time steps. If we extrapolate from the logarithmic trends at $n_{b}=$ $2.18\left(\delta_{R}=3.0\right)$ and $n_{b}=1.93\left(\delta_{R}=2.7\right)$ we find equivalent characteristic times of $10^{9}$ steps and $10^{14}$ steps, respectively. This indicates a very significant dependence on small changes in $n_{b}$. For a real material where one simulation time unit is around $10^{-7} \mathrm{~s}$, e.g., such as the example mentioned above of a film of $\simeq 1 \mu \mathrm{m}$ colloids, a reduction in repulsion length scale from $\delta_{R}=4$ to $\delta_{R}=2.7$ therefore generates an increase in the characteristic time for structural evolution from a fraction of a second to hundreds of days. Such a huge change in timescale indicates a strongly cooperative effect not simply related to the interaction between two isolated particles: indeed, logarithmic scaling in time is often associated with aging in complex energy landscapes such as labyrinths and glasses [5,19,20]. Such a sensitivity of timescale magnitude to system properties indicates a valuable control variable in the design of functional materials.

\section{CONCLUSIONS}

We have explored the complex interplay between shortrange attraction and long-range repulsion, focusing here on relatively strong repulsion, demonstrating effects on structure and kinetics that vary significantly depending on $E_{A}, \delta_{R}$, and $\Phi$. The effect of the repulsion is to drive separation of particles into "repulsion zones" of length scale $\delta_{R}$. The average number of particles that, by mass conservation, must nominally occupy each zone, $n_{b}$, combines the effects of repulsion length scale and particle area fraction and is a useful parameter to categorize structural and kinetic behavior. Ordered singleparticle systems give way, as $n_{b}$ increases above 1 , to chains driven and stabilized by repulsion, and the formation of a chain labyrinth. The formation of this labyrinth occurs at all $E_{A}$, and thermal coarsening is very slow even with zero attraction. As $n_{b}$ increases above $\simeq 2$, systems become characterized by mixtures of chains and compact groups. In this regime, kinetics of structural evolution (chains converting to groups under thermal fluctuation) is exceptionally strongly dependent on $n_{b}$. At yet higher $n_{b}$, systems demonstrate coexistence of different ordered and disordered structures which depend strongly on $E_{A}$ and persist for long times, again showing little 
sign of thermal coarsening. Finally at large $n_{b} \gg 1$ the system is dominated by repulsion, ultimately separating into layered or ordered compact groups. Short-length-scale structure and coarsening depend on $E_{A}$ but systems show similar power-law long-length-scale coarsening kinetics at all $E_{A}$.

Our results deepen our knowledge of the role of combined short-range attraction and long-range repulsion and the consequences of "frustrated" systems, demonstrating that an exceptionally rich range of out-of-equilibrium structures and mixed systems can be obtained and their aging toward equilibrium controlled. With recent developments in tuneable colloid chemistry such as "patchy" colloids, star copolymers, and so on, alongside tuneable interactions (strength and range, repulsive and attractive) from charge stabilization, magnetic interactions, and depletion attractions, results from this simple model potentially provide a road map for design of films and membranes with complex porous structure and aging properties at the colloidal length scale. That such rich behavior can arise from such a simple interaction underlines such materials' potential for novel and versatile functionality.

Here we have considered a relatively strong repulsion $E_{R}=45$ and focused on the roles of $\Phi$ and $\delta_{R}$, showing how these two parameters combine in $n_{b}$, and of $E_{A}$, exploring how attraction influences structure and kinetics in different regimes of $n_{b}$. Further work extending to higher $\Phi$, and varying the repulsion strength $E_{R}$ to providing a further tuning parameter to shift the balance between attraction and repulsion, is in progress and will be described elsewhere.

All data underpinning this publication are openly available from the University of Strathclyde KnowledgeBase [21].
[1] J. C. Berg, An Introduction to Interfaces and Colloids (World Scientific, Singapore, 2009).

[2] H. N. W. Lekkerkerker and R. Tuinier, Colloids and the Depletion Interaction (Springer, Dordrecht, 2011).

[3] J. Sabin, A. E. Bailey, and B. J. Frisken, Soft Matter 12, 5325 (2016).

[4] A. D. Dinsmore, P. L. Dubin, and G. M. Grason, J. Phys. Chem. B 115, 7173 (2011).

[5] Y. Zhuang and P. Charbonneau, J. Chem. Phys. 147, 091102 (2017).

[6] S. Merminod, T. Jamin, E. Falcon, and M. Berhanu, Phys. Rev. E 92, 062205 (2015).

[7] C. L. Klix, C. P. Royall, and H. Tanaka, Phys. Rev. Lett. 104, 165702 (2010).

[8] M. B. Sweatman and L. Lue, J. Chem. Phys. 144, 171102 (2016).

[9] S. V. Buldyrev, G. Malescio, C. A. Angell, N. Giovambattista, S. Prestipino, F. Saija, H. E. Stanley, and L. Xu, J. Phys.: Condens. Matter 21, 504106 (2009).

[10] A. B. de Oliveira, P. A. Netz, and M. C. Barbosa, Europhys. Lett. 85, 36001 (2009).
[11] M. C. Walters, P. Subramanian, A. J. Archer, and R. Evans, Phys. Rev. E 98, 012606 (2018).

[12] P. J. Camp, Phys. Rev. E 71, 031507 (2005).

[13] G. Malescio and G. Pellicane, Phys. Rev. E 70, 021202 (2004).

[14] M. D. Haw, Phys. Rev. E 81, 031402 (2010).

[15] A. T. Pham, Y. Zhuang, P. Detwiler, J. E. S. Socolar, P. Charbonneau, and B. B. Yellen, Phys. Rev. E 95, 052607 (2017)

[16] L. K. Tamm, editor, Protein-Lipid Interactions: From Membrane Domains to Cellular Networks (Wiley-VCH, Weinheim, Germany, 2005).

[17] N. Osterman, D. Babic, I. Poberaj, J. Dobnikar, and P. Ziherl, Phys. Rev. Lett. 99, 248301 (2007).

[18] Y. Shokef and T. C. Lubensky, Phys. Rev. Lett. 102, 048303 (2009).

[19] B. Reimann, R. Richter, and I. Rehberg, Phys. Rev. E 65, 031504 (2002).

[20] F. Krzyzewski and M. A. Zaluska-Kotur, Phys. Rev. E 77, 031502 (2008).

[21] https://doi.org/10.15129/69102f4a-fc08-472b-a562a9b2c8e0c770. 